\title{
Influência de programa de exercícios físicos na mobilidade funcional em idosos
}

\author{
Influence of a physical exercise program on functional mobility in the elderly \\ Influencia de un programa de ejercicio físico en la movilidad funcional del adulto mayor
}

Recebido: 09/11/2021 | Revisado: 18/11/2021 | Aceito: 22/11/2021 | Publicado: 02/12/2021

Thamy Ramana Moreira Prado ORCID: https://orcid.org/0000-0002-2167-1335 Universidade do Estado de Minas Gerais, Brasil E-mail: thamymprado@ hotmail.com

Juscelino de Souza Borges Neto ORCID: https://orcid.org/0000-0002-4551-3278 Universidade Federal de São João Del Rei, Brasil E-mail: celinoborges1980@gmail.com Larissa da Silva Lemos ORCID: https://orcid.org/0000-0002-8671-4500 Universidade do Estado de Minas Gerais, Brasil E-mail: larissalemos1@live.com

Juliana Mara Flores Bicalho

ORCID: https://orcid.org/0000-0003-1445-8234 Universidade Federal de São João Del Rei, Brasil E-mail: jmfbicalho@gmail.com

Gustavo Henrique Oliveira ORCID: https://orcid.org/0000-0003-2779-2549 Centro Universitário Una, Brasil E-mail: contato.gholiveira@gmail.com

Matheus Antônio Guimarães ORCID: https://orcid.org/0000-0002-9228-6725 Universidade Federal de São João Del Rei, Brasil E-mail: matheusjuridico@ hotmail.com

Patrícia Aparecida Tavares ORCID: https://orcid.org/0000-0002-3626-5217 Universidade Federal de São João Del Rei, Brasil E-mail: tavaresaguiar@yahoo.com.br

Rauno Álvaro de Paula Símola ORCID: https://orcid.org/0000-0001-8576-6934 Universidade do Estado de Minas Gerais, Brasil E-mail: rauno.simola@uemg.br

\begin{abstract}
Resumo
A mobilidade funcional pode ser descrita como a capacidade física e motora que um indivíduo dispõe para se locomover e realizar atividades cotidianas que permitam a sua autonomia; entretanto, o envelhecimento gradativo provoca a redução da mobilidade funcional, reduzindo a qualidade de vida do idoso e comprometendo sua saúde. O objetivo deste estudo é avaliar a influência de um programa de exercício físico na mobilidade funcional em idosos de um projeto de extensão da Universidade do Estado de Minas Gerais Unidade Divinópolis, descrevendo seus aspectos na mensuração do risco de quedas através do Time get up and go test (TUGT). Trata-se de um estudo analítico longitudinal tipo "antes e depois", em que foram registrados os dados de um grupo de indivíduos utilizando questionário específico e validado para essa finalidade. Esta pesquisa, que avaliou a mobilidade dos participantes antes e após um programa de exercício físico, utilizou o próprio participante como controle de si mesmo. Os participantes do estudo foram onze idosas com idade média de 66,81 anos $( \pm 6,18)$; todas do sexo feminino. Os principais resultados indicam que o comprometimento da mobilidade funcional agrava os impactos físicos sofridos pelo idoso mediante queda; aspectos sociais e ambientais podem motivar os idosos a se manterem em programas de exercícios físicos no longo prazo. Conclui-se que uma rotina consistente de exercícios físicos pode contribuir para a manutenção ou melhora da mobilidade funcional na terceira idade, considerando o fortalecimento do tecido muscular e do ganho de flexibilidade, que contribuem para uma melhor capacidade de equilíbrio e sustentação do corpo.
\end{abstract}

Palavras-chave: Exercício físico; Idoso; Acidentes por quedas.

\begin{abstract}
Functional mobility can be described as the physical and motor capacity that an individual has to move around and perform daily activities that allow their autonomy; however, gradual aging causes a reduction in functional mobility, reducing the quality of life of the elderly and compromising their health. The aim of this study is to evaluate the
\end{abstract}


influence of a physical exercise program on functional mobility in the elderly of an extension project of the State University of Minas Gerais Divinópolis Unit, describing its aspects in measuring the risk of falls through Time get up and go test (TUGT). This is a "before and after" longitudinal analytical study, in which data from a group of individuals were recorded using a specific and validated questionnaire for this purpose. This research, which evaluated the mobility of participants before and after a physical exercise program, used the participant as selfcontrol. Study participants were eleven elderly women with a mean age of 66.81 years $( \pm 6.18)$; all female. The main results indicate that the impairment of functional mobility aggravates the physical impacts suffered by the elderly through falls; social and environmental aspects can motivate the elderly to maintain long-term physical exercise programs. It is concluded that a consistent physical exercise routine can contribute to the maintenance or improvement of functional mobility in old age, considering the strengthening of muscle tissue and flexibility gain, which contribute to a better balance and support capacity of the body.

Keywords: Exercise; Aged; Accidental falls.

\section{Resumen}

La movilidad funcional puede describirse como la capacidad física y motora que tiene un individuo para moverse y realizar actividades diarias que le permitan su autonomía; sin embargo, el envejecimiento progresivo provoca una reducción de la movilidad funcional, reduciendo la calidad de vida de las personas mayores y comprometiendo su salud. El objetivo de este estudio es evaluar la influencia de un programa de ejercicio físico en la movilidad funcional en ancianos de un proyecto de extensión de la Unidad Divinópolis de la Universidad Estadual de Minas Gerais, describiendo sus aspectos en la medición del riesgo de caídas a través de Time get up and go prueba (TUGT). Se trata de un estudio analítico longitudinal "antes y después", en el que se registraron datos de un grupo de individuos mediante un cuestionario específico y validado para tal fin. Esta investigación, que evaluó la movilidad de los participantes antes y después de un programa de ejercicio físico, utilizó al participante como autocontrol. Los participantes del estudio fueron once mujeres mayores con una edad media de 66,81 años $( \pm 6,18)$; todas mujeres. Los principales resultados indican que el deterioro de la movilidad funcional agrava los impactos físicos que sufren las personas mayores a través de las caídas; Los aspectos sociales y ambientales pueden motivar a las personas mayores a mantener programas de ejercicio físico a largo plazo. Se concluye que una rutina de ejercicio físico consistente puede contribuir al mantenimiento o mejora de la movilidad funcional en la vejez, considerando el fortalecimiento del tejido muscular y la ganancia de flexibilidad, que contribuyen a un mejor equilibrio y capacidad de soporte del cuerpo.

Palabras clave: Ejercicio físico; Anciano; Accidentes por caídas.

\section{Introdução}

O aumento da expectativa de vida da população, consequentemente acarretou um crescimento da população idosa no mundo. Dessa forma, cresceram também a quantidade de estudos buscando investigar e conhecer melhor as condições de vida dessa população (Chagas et al., 2018). O processo de envelhecimento consta em inúmeras alterações moleculares em diferentes escalas. Essas alterações são causadas pela depreciação do índice de renovação e metabolismo celular, que por sua vez culminará na perda gradual das capacidades físicas e mentais do indivíduo (Organização Mundial da Saúde [OMS], 2018). De acordo com Souza et al. (2018), estas alterações podem ser inclusive cardíacas, vinculadas à diminuição da capacidade de esforço muscular do miocárdio para o ajuste dos batimentos solicitados pelo sistema nervoso autônomo.

Além disto, ocorre a maior propensão de desenvolvimento de patologias subordinadas a fatores genéticos, além do comprometimento de demais sistemas. Outras alterações ocorrem no sistema músculo esquelético, como encurtamentos nas fibras musculares assim como o comprometimento de sua capacidade elástica e em seu volume, perda de força e massa muscular, denominada sarcopenia, doença cujos sintomas são principalmente estabelecidos em indivíduos fisicamente inativos (Oliveira, 2009). Concomitantemente ocorre o ganho de gordura e de colágeno no tecido muscular, com uma redução de cerca de $15 \%$ de força muscular por década avançada. Isto demonstra a relevância da prática de exercícios físicos por idosos e a importância do desenvolvimento de pesquisas científicas que contribuam para a manutenção da qualidade de vida desta população (Greve et al., 2017). Gazolla et al. (2017) afirma que a prática de exercícios físicos em idosos melhora a qualidade de vida, além de reduzir o risco de acidentes por quedas.

Entende-se por mobilidade funcional, de acordo com Nascimento (2016) e Greve et al. (2017), a capacidade física e motora que um indivíduo dispõe para se locomover e realizar atividades cotidianas que permitam a sua autonomia. Logo, a 
redução da mobilidade funcional implica na restrição do potencial de deslocamento e na dependência de um terceiro indivíduo, seja ele um cuidador, enfermeiro ou profissional equivalente. A mobilidade funcional do idoso pode ser analisada no intuito de identificar e mensurar o risco de queda, tendo como resultado uma classificação que indica o nível de comprometimento da mobilidade e consequentemente a necessidade de intervenção, isto é, do suporte profissional qualificado.

Desta forma, a redução da mobilidade funcional, devido ao envelhecimento, está associada com a redução de massa muscular e óssea, e tende a elevar a possibilidade de quedas e lesões nesta parcela da população (Martinez et al., 2016; Siqueira et al., 2017). As quedas podem ainda comprometer (temporariamente ou permanentemente) a mobilidade, levando a contusões, fraturas, ou até mesmo ao óbito.

A prática de exercícios físicos na terceira idade tende a auxiliar na manutenção ou melhoria da capacidade e/ou mobilidade funcional, através do fortalecimento muscular e da mitigação da sarcopenia (Souza et al., 2017). De acordo com Soares et al. (2017), a realização de exercícios físicos na terceira idade mitiga o enfraquecimento dos músculos, reduz o nível de gordura no sangue e possibilita melhorias da flexibilidade e no equilíbrio estático ou dinâmico. É importante ainda considerar que a população idosa tem crescido gradativamente no Brasil e no mundo (Guadagnin, 2018), tornando esse estudo ainda mais relevante do ponto de vista social.

Diante disso, o objetivo deste estudo foi avaliar a influência de um programa de exercício físico na mobilidade funcional de idosos.

\section{Materiais e Métodos}

Trata-se de um estudo analítico longitudinal tipo "antes e depois", em que foram registrados os dados de um grupo de indivíduos utilizando questionário específico e validado para essa finalidade. Esta pesquisa, que avaliou a mobilidade dos participantes antes e após um programa de exercício físico, utilizou o próprio participante como controle de si mesmo (Hochman, et al. 2005).

Participaram dessa pesquisa todas as integrantes ( $\mathrm{n}=11$, idade média $=66,81 \pm 6,18$ anos) de um projeto de extensão da Universidade do Estado de Minas Gerais unidade Divinópolis intitulado "Vem pra Praça". Este projeto teve início em agosto de 2019 com o objetivo de proporcionar um programa de exercícios físicos para idosos com intuito de que essa população seja beneficiada nos aspectos psíquicos, físicos e sociais. Foram excluídos da amostra aqueles indivíduos que apresentaram doenças cardíacas, hipertensão arterial crônica ou prótese em membros inferiores.

$\mathrm{O}$ instrumento de coleta de dados utilizado foi o teste Time get up and go test (TUGT) que tem objetivo de avaliar mobilidade funcional idosos durante a marcha (Podsiadlo \& Richardson, 1991). A coleta de dados foi realizada em dois momentos distintos. A primeira coleta de dados foi realizada em agosto de 2019 (Pré-intervenção) e a segunda coleta de dados foi realizada em dezembro de 2019 (Pós-intervenção), após um período de treinamento físico. Em ambas as coletas, todas as voluntárias foram submetidas ao teste TUGT. Foram coletados no momento pré-intervenção, os dados das participantes como data de nascimento.

Para a realização dos testes TUGT foram utilizados uma cadeira com 45 centímetros de largura de assento e 48 centímetros de altura, com apoios laterais e base fixa; um cronômetro; fita adesiva para delimitar o trajeto e uma trena de medição. Foi orientado e certificado o entendimento dos passos a serem aplicados, demonstrando o procedimento uma vez. Para a realização do teste, é preciso que o participante inicie sentado, e aguarde o sinal do orientador TUGT para que possa se levantar, evitando se apoiar no suporte da cadeira, e que caminhe três metros retilineamente. Chegando ao final da marcação, o paciente se vira em $180^{\circ}$ de forma a retornar ao ponto de partida, e sentar-se novamente. Se necessário, é feita a correção de movimentos errôneos ou incompletos, reforçando pontos importantes, tais como: chegar até a marca no chão e sentar-se 
encostando completamente o tronco no encosto da cadeira. Caso o idoso apresente alguma dificuldade de entendimento (ou esquecimento), que o faça interromper o percurso, refaça a orientação a respeito da forma correta de execução e reinicie o teste. Caso o participante faça qualquer pergunta durante o teste, como por exemplo: "É para sentar?", responda: "Faça como eu lhe disse para fazer". É permitido ao participante o uso de dispositivo de auxílio à marcha, como bengala, ou andador. O participante deve estar usando seu sapato habitual. Para cronometrar o tempo: o cronômetro deve-se ser disparado quando o participante projetar os ombros à frente desencostando da cadeira, e deve ser parado, quando o mesmo encostar completamente o tronco no encosto da cadeira. O tempo foi marcado a partir da projeção do tronco das participantes para frente, no sentido de levantar-se, e finalizado quando as participantes se sentaram apoiando o tronco em toda a sua extensão no encosto da cadeira. Para os tempos cronometrados considera-se: inferior a 10 segundos - normal/baixo risco de queda; entre 10 e 20 segundos médio/moderado risco de queda; superior a 20 segundos - elevado risco de queda. Segundo a literatura, o tempo acima de 12,4 segundos indica risco aumentado para quedas (Podsiadlo \& Richardson, 1991).

A intervenção se deu através de um programa de exercícios físicos aplicado por 3 meses, 2 vezes por semana, 1 hora por dia, na praça Nelson Pelegrino, bairro Bom Pastor da cidade de Divinópolis - MG, de 18:30 às 19:30 horas às segundas e quartas-feiras. As atividades foram planejadas por duas acadêmicas do curso de Educação Física em conjunto com os docentes orientadores da UEMG.

As atividades foram divididas em quatro momentos: aquecimento, exercícios físicos e relaxamento. Foram feitas atividades do estilo funcional, dança e musculação utilizando os aparelhos de academia ao ar livre que possuíam na praça. Ao início das atividades eram realizadas caminhas/trotes de 5 minutos em volta da praça. Nas segundas-feiras eram feitas atividades com dança e alongamentos. Nas quartas-feiras os exercícios eram feitos com os aparelhos de academia que possuíam na praça. Exercícios estes onde todos os grupos musculares eram treinados, em formato de circuito com 15 séries. A intervenção foi planejada, estruturada e programada também pelas acadêmicas em conjunto aos docentes orientadores do curso de Educação Física Bacharelado da UEMG unidade Divinópolis, ambos de forma voluntária.

Os dados foram tabulados e analisados no software estatístico IBM® SPSS® versão 22.0 para Windows e foi adotado um nível de significância de $\mathrm{p}<0,05$. Pelo teste de normalidade Kolmogorov-Smirnov, os resultados do TUGT apresentaram normalidade e foram demonstrados por média e desvio padrão. As médias foram comparadas por teste $t$ Student de duas amostras pareadas.

Este estudo foi realizado em conformidade com todas as normas de pesquisa em seres humanos e foi aprovado pelo Comitê de Ética em Pesquisa da Universidade do Estado de Minas Gerais (UEMG) sob parecer nº 3.679.336.

\section{Resultados e Discussão}

O desempenho no teste TUGT e a classificação de queda antes e após o programa de treinamento é descrito na tabela 1. Foi observada uma melhora estatística do desempenho no teste TUGT após o programa de treinamento $(10.22+1.82 \mathrm{~s}$ vs. $9.04+0.88 \mathrm{~s} ; \mathrm{p}<0.05)$. 
Tabela 1 - Desempenho no teste TUGT e classificação do risco de queda antes e após o programa de treinamento.

\begin{tabular}{ccccc}
\hline Voluntária & $\begin{array}{c}\text { Desempenho } \\
\text { TUGT Pré (s) }\end{array}$ & $\begin{array}{c}\text { Classificação do risco de queda } \\
\text { Pré }\end{array}$ & \multicolumn{2}{c}{$\begin{array}{c}\text { Desempenho } \\
\text { TUGT Pós (s) }\end{array}$} \\
\hline A & 9.75 & Normal & 9.38 & Normal \\
B & 9.56 & Normal & 8.66 & Normal \\
C & 11.02 & Risco Moderado de Queda & 9.52 & Normal \\
D & 9 & Normal & 8.36 & Normal \\
E & 8.59 & Normal & 8.57 & Normal \\
F & 10.66 & Risco Moderado de Queda & 8.96 & Normal \\
G & 9.50 & Normal & 9.46 & 7.68 \\
H & 9 & Normal & 8.26 & Normal \\
I & 9 & Normal & 9.81 & 10.88 \\
J & 11.38 & Risco Moderado de Queda & Normal \\
K & 15 & Risco Moderado de Queda & & Risco Moderado de Queda \\
\hline Média & $10.22(1.82)$ & & $9.04(0.88) *$
\end{tabular}

DP: desvio padrão; $* \mathrm{p}=0.00662$. Fonte: Autores $(2021)$.

No momento pré-intervenção, 63,63\% das participantes do estudo apresentaram um tempo de realização do teste TUGT abaixo de $10 \mathrm{~s}$, indicando um baixo risco de quedas. Após o programa de treinamento, 36,37\% das participantes apresentaram um tempo de realização do teste TUGT entre 10 e $20 \mathrm{~s}$, indicando um risco de quedas moderado.

Um total de $90,9 \%$ das participantes registraram um tempo inferior a 10 segundos na pós-intervenção. Nesse momento de medida, uma única participante não conseguiu a marca de baixo risco de queda, e ainda sim obteve uma melhora, isto é, uma redução de $27,46 \%$ no tempo registrado no TUGT.

Os resultados aqui apresentados estão em concordância com os descritos por Guadagnin (2018), que demonstrou que a junção de exercícios físicos aeróbicos e anaeróbicos em idosos permite a melhora no funcionamento do sistema respiratório, além de proporcionar otimização do condicionamento físico, ganho de força e capacidade de contração muscular.

No estudo de Fernandes et al. (2012), onde oito idosos (62,5\% mulheres) participaram de um programa de exercícios físicos na cidade de Curitiba - PA, em período análogo; foi notado que um programa de exercícios físicos sendo eles, treino de força, equilíbrio e propriocepção traz uma melhora na mobilidade funcional de idosos, medida através do TUGT ( $p=0,003$ ).

De acordo com Wingerter et al. (2020), de 28 a 45\% dos idosos (neste caso acima de 65 anos) no Brasil sofrem quedas todos os anos e, destes, de 5 a $10 \%$ possuem a evolução do quadro clínico para óbito; os pesquisadores identificaram grandes variações no perfil epidemiológico de óbitos por queda, entretanto a restrição da mobilidade funcional foi item de destaque. A redução da mobilidade funcional demonstrou-se intimamente ligada com o sedentarismo e indicou que não somente a mobilidade é um fator de complicação, mas igualmente a sarcopenia, pois expõe a estrutura óssea e agrava as consequências do impacto, sendo congruente com as abordagens realizadas neste artigo.

Neste mesmo viés, Pontes et al. (2017) descrevem a importância do envelhecimento ativo para a redução dos casos de internação e depressão em idosos. Em seus estudos, 36 participantes de ambos os sexos (72,5\% mulheres) de 63 a 84 anos, participaram de um programa de exercícios por meio do método Pilates durante 10 semanas, na cidade de Salvador - BA; foram identificadas melhorias expressivas no quesito de ganho de flexibilidade, resistência muscular, redução da sensação de dores nas atividades cotidianas e melhoria na mobilidade funcional/equilíbrio, utilizando também como métrica o TUGT. Os mesmos autores ainda reforçam que o grande motivador para a adesão ao programa foi a oportunidade da participação coletiva e a preocupação com quedas, assim como nos estudos de Amorim (2016).

Os achados dos estudos citados contribuem e se posicionam a favor dos dados coletados no presente estudo, tendo em vista que todos os artigos aqui citados, excluíram de suas amostras idosos com doenças crônicas adquiridas/não transmissíveis, e demonstraram em diferentes esferas as contribuições dos exercícios físicos regulares para a manutenção da mobilidade funcional para idosos. 
Já Lacerda et al. (2018), analisaram oito idosos residentes em duas Instituições de Longa Permanência (ILPI) no município de Betim - MG, de ambos os sexos. Estes idosos participaram de um programa de exercícios físicos durante um período de 18 semanas, e realizaram o TUGT antes e posteriormente a intervenção. Entretanto, 50\% dos participantes não apresentaram melhoras significativas do início ao tempo médio do programa, e dois participantes apresentaram um desempenho abaixo do primeiro tempo cronometrado, aparentemente em função de iatrogenia (efeitos diversos de tratamentos médicos) e/ou dinapenia (redução da força muscular não causada por patologia).

Estes resultados corroboram com Valduga (2018), em que 10 idosos (70\% mulheres) participaram de um programa de exercícios físicos com auxílio/estímulo de um jogo virtual na cidade de Taguatinga-DF, durante o mesmo período. Seis idosos apresentaram uma piora (na segunda etapa) no desempenho do TUGT, aparentemente devido a uma rotina de exercícios que solicitavam a flexão e extensão dos membros inferiores. O que culminou em desconforto no complexo articular, indicando possível osteoartrite, comumente diagnosticada em idosos longevos.

\section{Considerações Finais}

O processo gradativo de envelhecimento traz desafios no que tange a manutenção da mobilidade funcional, pois afeta desde o processo cognitivo ao metabólico, e musculoesquelético. Mediante o objetivo proposto, de avaliar a influência de um programa de exercício físico na mobilidade funcional em idosos descrevendo seus aspectos na mensuração do risco de quedas através do teste TUGT; foi possível identificar que para a amostra analisada, o programa regular de atividades físicas influenciou positivamente na redução global do risco de quedas nas idosas participantes do projeto "Vem Pra Praça".

Considerando a pesquisa realizada é sugerido um programa de treinamento resistido em que todos os grupos musculares sejam treinados, juntamente com exercícios do estilo funcionais e alongamentos. Exercícios esses a serem realizados pelo menos duas vezes na semana com duração de uma hora, para uma melhora significativa na mobilidade funcional de idosos.

\section{Referências}

Amorim, D. N. P. (2016). Associação da capacidade funcional com o perfil epidemiológico de idosos longevos. [Dissertação de Mestrado, Universidade Católica de Brasília]. https://bdtd.ucb.br:8443/jspui/handle/tede/2183

Chagas, D. L., Rodrigues, A. L. P., Brito, L. C., \& Soares, E. S. (2018). Relação entre o equilíbrio corporal e o risco de quedas em idosos de um projeto social de Fortaleza-CE. RBPFEX - Revista Brasileira De Prescrição E Fisiologia Do Exercício, 12(76), 547-555. http://www.rbpfex.com.br/index.php/rbpfex/article/view/1455

Fernandes, A. M. B. L., Ferreira, J. J. A., Stolt, L. R. O. G., Brito, G. E. G., Clementino, A. C. C. R., \& Sousa, N. M. (2012). Efeitos da prática de exercício físico sobre o desempenho da marcha e da mobilidade funcional em idosos. Fisioter Mov, 25(4), 821-830. https://doi.org/10.1590/S0103-51502012000400015

Galloza, J., Castillo, B., \& Micheo, W. (2017). Benefits of Exercise in the Older Population, Physical Medicine and Rehabilitation Clinics of North America, 28(4), 659-669 https://doi.org/10.1016/j.pmr.2017.06.001

Greve, P., Guerra, A., Portela, M., Portes, M., \& Rebelatto, J. (2017). Correlações entre mobilidade e independência funcional em idosos institucionalizados e não-institucionalizados. Fisioterapia em Movimento, 20(4). https://periodicos.pucpr.br/index.php/fisio/article/view/18969

Guadagnin, E. C. (2018). Mobilidade funcional em idosos: influência de parâmetros musculares e de treinamento. [Tese de Doutorado, Universidade Federal do Rio Grande do Sul]. http://hdl.handle.net/10183/181830

Hochman, B., Nahas F. X., Oliveira Filho, R. S., \& Ferreira L. M. (2005). Desenhos de pesquisa. Acta Cir Bras, 20(2), 02-9. https://doi.org/10.1590/S010286502005000800002

Lacerda, T. T. B., Cruz, T. K. F., Souto, D. O., Souza, A. C. L., Alckin, J. P., \& Gonçalves, R. V. (2018). Efeito da reabilitação utilizando o videogame Nintendo Wii no equilíbrio de idosos institucionalizados: um estudo experimental de caso único. Revista NBC, 8(15). https://www.metodista.br/revistas/revistas-izabela/index.php/bio/article/view/1742

Martinez, B. P., Santos, M. R., Simões, L. P., Ramos, I. R., Oliveira, C. S., Forgiarini Júnior, L. A., Camelier, F. W. R., \& Camelier, A. A. (2016). Segurança e reprodutibilidade do teste timed up and go em idosos hospitalizados. Rev Bras Med Esporte, 22(5), 408-411. https://doi.org/10.1590/1517869220162205145497 
Nascimento, C. F. (2016). Determinantes sociais da mobilidade funcional e quedas em idosos do município de São Paulo: uma análise multinível. [Dissertação de Mestrado, Universidade de São Paulo]. https://doi.org/10.11606/D.6.2016.tde-23052016-134527

Oliveira, S. F. (2009). Impacto do fortalecimento muscular na reeducação da marcha de idosos institucionalizados. Fisioterapia Brasil. 10(1). https://portalatlanticaeditora.com.br/index.php/fisioterapiabrasil/article/view/1493

Organização Mundial da Saúde (OMS). Folha informativa - Envelhecimento e saúde. 2018. https://www.paho.org/bra/index.php?option=co m_content\&view=article\&id=5661:folha-informativa-envelhecimento-e-saude \&Itemid=820

Pontes, S. S., Moinhos, C., Andrade, J. A., Schulz, R., Bouzas, M., Doria, L., \& Guedes, J. M. (2017). Perfil e mobilidade funcional em idosos. Revista Intercâmbio. 9, 95-110. http://www.intercambio.unimontes.br/index.php/intercambio/article/view/172

Podsiadlo, D., \& Richardson, S. (1991). The timed "Up \& Go": A test of basic functional mobility for frail elderly persons. Journal of the American Geriatrics Society, 39(2), 142-148. https://doi.org/10.1111/j.1532-5415.1991.tb01616.x

Siqueira, F. V., Facchini, L. A., Piccini, R. X., Tomasi, E., Thumé, E., Silveira, D. S., Vieira, V., \& Hallal, P. C. (2017). Prevalência de quedas em idosos e fatores associados. Rev Saude Publica. 41(5), 749-56. https://doi.org/10.1590/S0034-89102007000500009

Soares, A. V., Marcelino, E., Maia, K. C., \& Borges Junior, N. G. (2017). Relação entre mobilidade funcional e dinapenia em idosos com fragilidade. Einstein, 15(3), 278-282. https://doi.org/10.1590/S1679-45082017AO3932

Souza, L. H. R., Brandão, J. C. S., Fernandes, A. K. C., \& Cardoso, B. L. C. (2017). Queda em idosos e fatores de risco associados. Revista de Atenção à Saúde, 15(54), 55-60. https://doi.org/10.13037/ras.vol15n54.4804

Souza, M. M., Ansai, J. H., Vilarinho, A. C., \& Andrade, L. P. (2018). Teste TUG é um bom preditor de declínio funcional para idosos com comprometimento cognitivo leve (CCL) e doença de Alzheimer (DA) na fase leve? Um estudo longitudinal de 32 meses. UFSCar São Carlos XXV CIC e X CIDTI - 2018. http://www.copictevento.ufscar.br/index.php/ictufscar2018/saocarlos-2018/paper/view/975

Valduga, L. V. A. (2018). Efeito de uma intervenção breve com o uso de jogo virtual em idosos longevos. [Dissertação de Mestrado, Universidade Católica de Brasília]. https://bdtd.ucb.br:8443/jspui/handle/tede/2447

Wingerter, D. G., Barbosa, I. R., Moura, L. K. B., Maciel, R. F., \& Alves, M. S. C. F. (2020). Mortalidade por queda em idosos: uma revisão integrativa. Revista Ciência Plural, 6(1), 119-136. https://doi.org/10.21680/2446-7286.2020v6n1ID18366 